Article

\title{
Multipoles of Even/Odd Split-Ring Resonators
}

\author{
Andrew Chen, Ashok Kodigala, Thomas Lepetit and Boubacar Kanté * \\ Department of Electrical and Computer Engineering, University of California San Diego, La Jolla, \\ CA 92093-0407, USA; E-Mails: anc067@ucsd.edu (A.C.); akodigala@eng.ucsd.edu (A.K.); \\ tlepetit@eng.ucsd.edu (T.L.) \\ * Author to whom correspondence should be addressed; E-Mail: bkante@ucsd.edu; \\ Tel.: +1-858-822-1658; Fax: +1-858-534-1225.
}

Received: 28 June 2015 / Accepted: 28 July 2015 / Published: 5 August 2015

\begin{abstract}
The ultimate goal of metamaterial engineering is to have complete control over the electromagnetic constitutive parameters in three-dimensional space. This engineering can be done by considering either single meta-atoms or full meta-arrays. We follow the first route and perform numerical simulations of split-ring resonators, with different gap numbers and under varying illumination scenarios, to investigate their individual multipolar scattering response. For the fundamental resonance, we observe that odd-gap rings always exhibit overlapping electric and magnetic dipole responses while even-gap rings only exhibit that behavior accidentally. We expect our results to foster progress in the engineering of three-dimensional disordered metamaterials.
\end{abstract}

Keywords: metamaterial; multipole; symmetry; random

\section{Introduction}

In the last fifteen years, intense efforts have been devoted to the investigation of man-made artificial materials or metamaterials [1,2]. Metamaterials are attractive because they possess a rich variety of electromagnetic constitutive parameters, such as electric permittivity, magnetic permeability, and bianisotropy, all of which can be tailored by their geometry [3-5]. For example, metamaterials have made transformation optics possible by providing a blueprint for the realization of anisotropic, inhomogeneous, and graded electromagnetic properties [6,7]. To date, almost all metamaterials investigated have been periodic. Indeed, periodicity not only simplifies the simulation of metamaterials but also their fabrication. However, at optical frequencies, current top-down fabrication technologies, 
such as electron-beam lithography, are very costly, intrinsically planar, and not compatible with large scale-devices. Bottom-up fabrication technologies could alleviate part of this problem but suffer from limitations of their own, namely the absence of long-range order [8], even though they've made strides in recent years [9]. Therefore, to leverage bottom-up fabrication technologies and go towards three-dimensional, large-scale devices, it is necessary to develop novel tools for a thorough understanding of disordered metamaterials $[10,11]$.

Metamaterials electromagnetic properties have most often been investigated using parameter retrieval, i.e., they were computed from reflection and transmission coefficients of periodic arrays [12]. However, this approach is no longer possible for disordered metamaterials. To overcome this difficulty, another approach to metamaterials is needed. For instance, metamaterials can be seen as a collection of essential building blocks, also referred to as meta-atoms, which support different multipoles: electric dipoles, magnetic dipoles, as well as higher-order multipoles [13]. From this perspective, it is the induction of this microscopic polarization and magnetization that gives rise to macroscopic effective properties, such as electric permittivity, magnetic permeability, or bianisotropy [14]. Then, a natural and versatile method of considering the electromagnetic properties of disordered metamaterials consists of computing the intrinsic multipoles of meta-atoms and complement those with a mixing law [15]. Investigations of individual meta-atoms thus represent a required stepping stone towards the engineering of disordered metamaterials.

Split-ring resonators are one of the essential building blocks of metamaterials. They have been used in the first experimental demonstrations to obtain negative permeability and negative index and, more recently, in magnetic resonance imaging and cloaking [16,17]. The first investigations of one-gap split-ring resonators were essentially focused on their fundamental resonance due to their resonant magnetic response. It was referred to as an LC resonance, in analogy with electrical engineering practice, because the gap and ring were seen as a capacitance (C) and inductance (L), respectively [18]. With the transition to optics, however, it was soon realized that all resonances can be interpreted within the unified framework of plasmonic, standing-wave resonances [19,20]. In such models, splitrings are essentially viewed as thin plasmonic films that are truncated into a wire and bent into a splitring [21]. Other investigations focused on the properties of multi-gap split-ring resonators and their coupling effects [22], most notably to extend the operating range of one-gap split-rings because of its saturation-imposed limits at optical frequencies [23]. A similar plasmonic, standing-wave picture was shown to apply to multi-gap split-rings as well [24]. In addition, it was recently shown that one-gap split-ring resonators are possible platforms for the realization of random metamaterials with a negative index due to the inherent superposition of their intrinsic electric and magnetic dipoles [25].

In this paper, we go one step further and investigate the multipoles of split-ring resonators with an odd or even number of gaps (from one to four gaps) and show that electric and magnetic dipoles are always degenerate in the odd case, while that is only accidentally true in the even case. We present extensive numerical evidence to support this claim, namely multipole coefficients of individual split-ring resonators retrieved from full-wave simulations, and thus shed light on the effective properties of disordered metamaterials. 


\section{Multipolar Decomposition}

We first consider a one-gap split-ring resonator (1-SRR) made of gold and surrounded by free space, with a length $L=700 \mathrm{~nm}$, width $W=50 \mathrm{~nm}$, thickness $T=50 \mathrm{~nm}$, and gap $G=200 \mathrm{~nm}$ (see Figure 1a). We illuminate this 1-SRR with a plane wave for three different incidences and/or polarizations. In case 1, the electric field is in-plane and along the gap and the magnetic field is out-of-plane (see Figure 1b). In case 2, the electric field is in-plane but normal to the gap and the magnetic field is out-of-plane (see Figure 1c). In case 3, both the electric and magnetic fields are in-plane and the electric field is along the gap while the magnetic field is normal to it (see Figure 1d). These cases were chosen because the fundamental resonance of the 1-SRR is excited in all three cases [20].

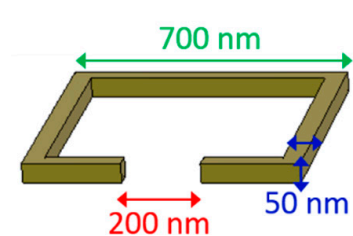

(a)
Case 1

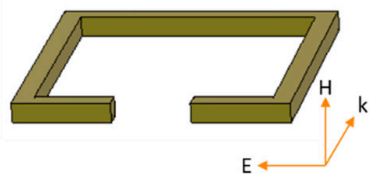

(b)
Case 2

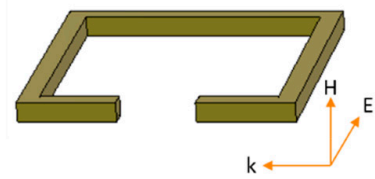

(c)
Case 3

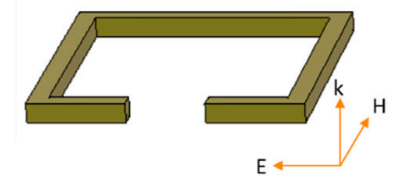

(d)

Figure 1. (a) Dimensions of the 1-SRR: $\mathrm{L}=700 \mathrm{~nm}, \mathrm{~W}=50 \mathrm{~nm}, \mathrm{~T}=50 \mathrm{~nm}$, and $\mathrm{G}=200 \mathrm{~nm}$ (b) Case 1: electric field in-plane and along the gap, magnetic field out-of-plane (c) Case 2: electric field in-plane and normal to the gap, magnetic field out-of-plane (c) Case 3: electric and magnetic fields in-plane, along the gap and normal to it, respectively. All the gold SRRs are described using the Drude model with a plasma frequency $\left(\omega_{\mathrm{p}}=1.367 \times 10^{16} \mathrm{rad} / \mathrm{s}\right)$ and collision frequency $\left(\omega_{\mathrm{c}}=6.478 \times 10^{13} \mathrm{rad} / \mathrm{s}\right) \mathrm{based}$ on values from [31].

The scattered electric field (total field minus incident field) is recorded in the near-field on a virtual sphere completely enclosing the 1-SRR. In spherical coordinates, the scattered electric field can be compactly expressed in terms of vector spherical harmonics (VSH), $\mathbf{N}_{n m}$ and $\mathbf{M}_{n m}[26,27]$

$$
E_{s c a}(r, \theta, \varphi)=\sum_{n=1}^{\infty} \sum_{m=-n}^{+n} k^{2} E_{n m}\left[a_{n m} N_{n m}(r, \theta, \varphi)+b_{n m} M_{n m}(r, \theta, \varphi)\right]
$$

where $n$ and $m$ are indices characterizing the zenithal and azimuthal dependence of the VSHs, $k$ is the wavevector, and $E_{n m}$ a scaling coefficient. Since the VSHs are orthogonal to each other, we can compute the expansion coefficients $a_{n m}$ and $b_{n m}$ by projection and appropriate normalization.

$$
\begin{aligned}
& a_{n m}=\frac{\int_{0}^{2 \pi} \int_{0}^{\pi} \mathbf{E} \cdot \mathbf{N}_{n m}^{*} \sin \theta d \theta d \varphi}{\int_{0}^{2 \pi} \int_{0}^{\pi} \mathbf{N}_{n m} \cdot \mathbf{N}_{n m}^{*} \sin \theta d \theta d \varphi} \\
& b_{n m}=\frac{\int_{0}^{2 \pi} \int_{0}^{\pi} \mathbf{E} \cdot \mathbf{M}_{n m}^{*} \sin \theta d \theta d \varphi}{\int_{0}^{2 \pi} \int_{0}^{\pi} \mathbf{N}_{n m} \cdot \mathbf{M}_{n m}^{*} \sin \theta d \theta d \varphi}
\end{aligned}
$$

The scattering cross-section of the 1-SRR is then simply expressed as 


$$
C_{s c a}=k^{2} \sum_{n=1}^{\infty} \sum_{m=-n}^{+n} n(n+1)\left[\left|a_{n m}\right|^{2}+\left|b_{n m}\right|^{2}\right]
$$

Furthermore, we can relate these expansion coefficients to Cartesian multipoles [26], most notably the lowest-order multipoles (electric dipole, $\mathbf{p}$, magnetic dipole, $\mathbf{m}$, and electric quadrupole, $\mathbf{Q}$ ). Finally, we can use these to decompose the scattering cross-section

$$
C_{s c a}=C_{s c a}^{p}+C_{s c a}^{m}+C_{s c a}^{Q}+\cdots
$$

Note that in the limit of geometrical optics, and accounting for the extinction paradox [27], the scattering cross-section is bounded by twice the geometrical cross-section $\left(C_{s c a} \leq 0.98 \mu \mathrm{m}^{2}\right)$.

In the following, we use this decomposition to identify the multipolar character of each resonance in all three cases of interest (see Figure 1). Besides, we restrict to the four lowest order Cartesian multipoles with the strongest response, namely electric dipoles, $p_{x}$, and, $p_{y}$, magnetic dipole, $m_{z}$, and electric quadrupole, $Q_{x y}$.

\section{From 1-SRR to 4-SRR}

We plot in Figure 2 the different scattering cross-sections (electric dipole, magnetic dipole, electric quadrupole, and total) of the 1-SRR for the three different cases defined in Figure 1. In case 1, only modes that are even with respect to the $x y$-plane and odd with respect to the $x z$-plane can be excited. In case 2, only the former symmetry holds true, while in case 3, only the latter symmetry holds true. Due to these symmetry constraints, the electric dipole, $p_{x}$, cannot be excited in cases 1 and 3 . As can be seen in Figure 2, in all three cases there are two resonances that are always excited $\left(\mathrm{f}_{1}=51 \mathrm{THz}\right.$, $\left.\mathrm{f}_{3}=151 \mathrm{THz}\right)$ while in the second case there is also an additional resonance $\left(\mathrm{f}_{2}=106 \mathrm{THz}\right)$. Resonance 2 is due to the excitation of the electric dipole, $p_{x}$. Resonances 1 and 3 can be excited either by the electric field along the gap, or the magnetic field normal to the plane of SRR, or a combination of both, and yield both an electric dipole, $p_{y}$, and a magnetic dipole, $m_{z}$, response. In addition, resonance 3 shows an in-plane quadrupole, $Q_{x y}$, response. Surprisingly, when both fields are present with the right polarization (case 1), their effects do cumulate for resonance 1 but not for resonance 3. This would seem to indicate that case 3 is the most favorable to multipole overlap but, in a planar configuration, the magnetic dipole, $m_{z}$, does not radiate in the direction of propagation, thus making case 1 the preferred illumination scenario.

We plot in Figure 3, the scattering cross-sections of the 2-SRR (even) for the three different cases defined in Figure 1. We highlight that all further additions of gaps carry the same dimensions of $G=200 \mathrm{~nm}$ centered along a given side of the starting square split-ring (see Figure 1a). In case 1, only modes that are even with respect to the $x y$-plane and odd with respect to the $x z$-plane can be excited. In case 2 , only modes that are even with respect to the $x y$-plane and odd with respect to the $y z$-plane can be excited. In case 3, only modes that are even with respect to the $y z$-plane and odd with respect to the $x z$-plane can be excited. Due to these symmetry constraints, the electric dipole, $p_{x}$, cannot be excited in cases 1 and 3, the electric dipole, $p_{y}$, cannot be excited in case 2, and the magnetic dipole, $m_{z}$, and electric quadrupole, $Q_{x y}$, cannot be excited in case 3 . As a result, there can only be an overlap between the electric dipole, $p_{y}$, and the magnetic dipole, $m_{z}$, in case 1 . However, as can be seen from Figure 3, both modes are only weakly excited in this case $\left(f_{1}=98 \mathrm{THz}\right)$. Unlike the 1-SRR, 
in case 2, the 2-SRR has a small overlap between the electric dipole, $p_{x}$, and the magnetic dipole, $m_{z}$, ( $f_{1}=98 \mathrm{THz}, f_{2}=106 \mathrm{THz}$ ), which could prove useful in the design of novel metamaterials. This overlap is not due to symmetry but rather to the specific dimensions of the 2-SRR considered here and, hence, is accidental. For the 2-SRR, case 2 is the preferred illumination scenario.

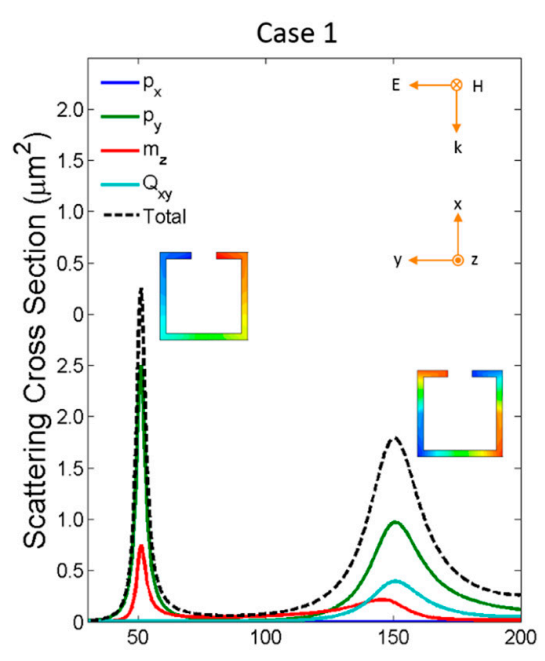

(a)

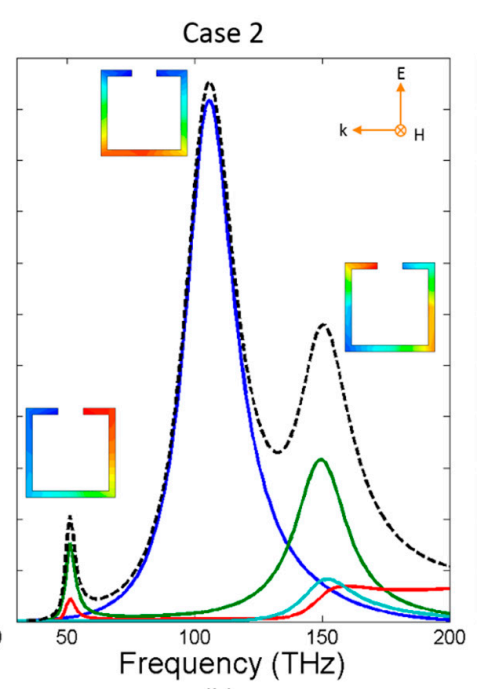

(b)

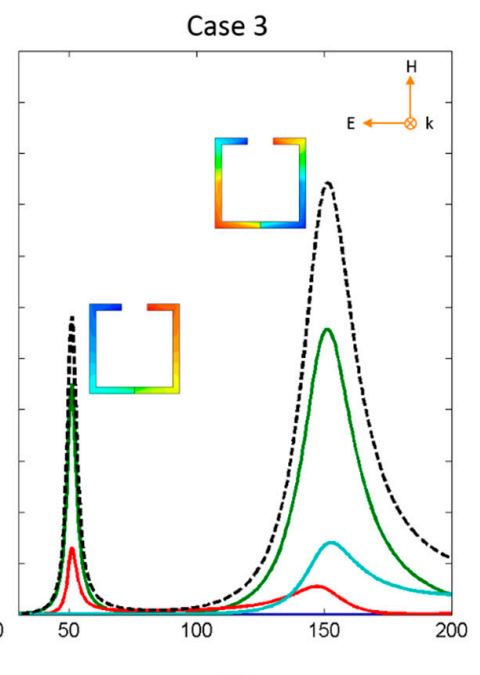

(c)

Figure 2. Multipolar decomposition of the scattering cross-section, for a 1-SRR between $30 \mathrm{THz}$ and $200 \mathrm{THz}$, into the lowest order multipoles; (a) Case 1: electric field in-plane and along the gap, magnetic field out-of-plane; two resonances are observed at $f_{1}=51 \mathrm{THz}$ and $f_{3}=151 \mathrm{THz}$ (b) Case 2: electric field in-plane and normal to the gap, magnetic field out-of-plane; three resonances are observed, those observed in case 1 plus another one at $\mathrm{f}_{2}=106 \mathrm{THz}$ due to the excitation of the electric dipole, $p_{x}$ (c) Case 3: electric and magnetic fields in-plane, along the gap and normal to it, respectively; two resonances are observed, just as in Case 1. The resonances excited in Case 1 and 3 yield both an electric dipole, $p_{y}$, and a magnetic dipole, $m_{z}$, response. Resonance 3 also shows an in-plane quadrupole, $Q_{x y}$, response in all cases. Insets show the real part of $E_{z}$ at the corresponding resonance frequencies using a rainbow colormap ( $\min$ in blue, zero in green, and max in red).

In Figure 4, we plot the scattering cross-sections of the 3-SRR (odd) for the three cases defined in Figure 1. The 3-SRR has exactly the same symmetry properties as the 1-SRR, thus exactly the same multipoles are forbidden or allowed. As can be seen in Figure 4, in all three cases there are two resonances that are always excited $\left(f_{1}=121 \mathrm{THz}, \mathrm{f}_{2}=219 \mathrm{THz}\right)$ and, similar to the 1-SRR, when both fields are present with the right polarization (case 1), their effects do cumulate for resonance 1 but not for resonance 2. Furthermore, just as for the 1-SRR, the planar configuration of case 3 is not suitable for multipole overlap as it suffers from a lack of radiation along the direction of propagation. A notable difference for the 3 -SRR is that resonance 2 has a much less prominent electric quadrupole, $Q_{x y}$, than the 1-SRR. Overall, the 3-SRR is very similar to the 1-SRR, e.g., case 1 is the preferred illumination scenario, with the only important difference being that its resonances occur at higher frequencies. 


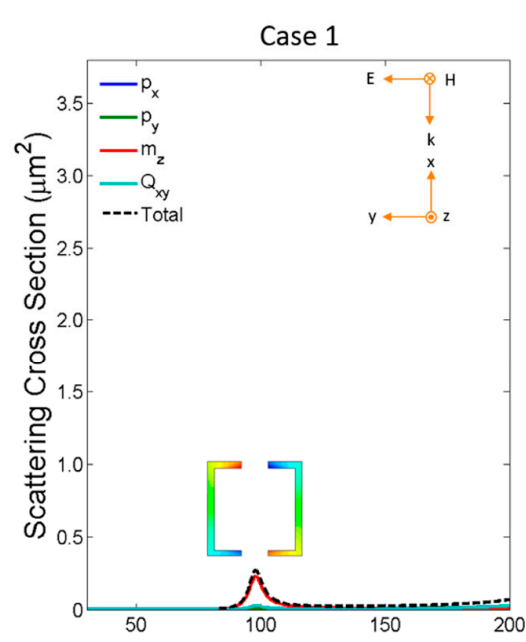

(a)

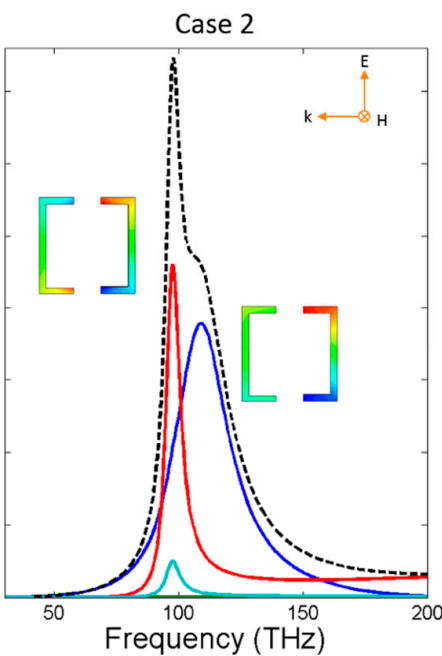

(b)

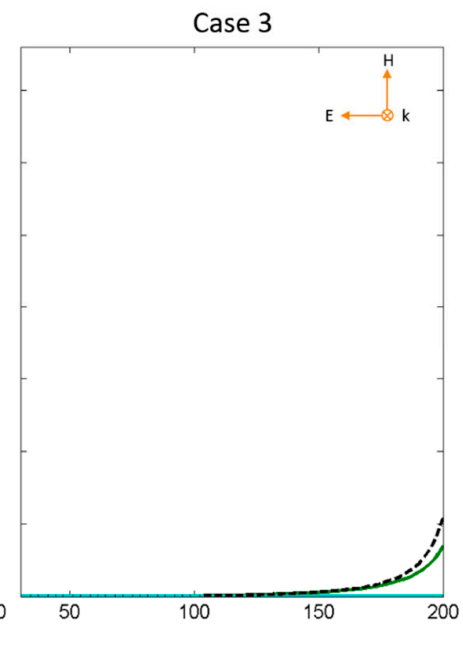

(c)

Figure 3. Multipolar decomposition of the scattering cross-section, for a 2-SRR between $30 \mathrm{THz}$ and $200 \mathrm{THz}$, into the lowest order multipoles; (a) Case 1: electric field in-plane and along the gaps, magnetic field out-of-plane; one resonance is observed at $f_{1}=98 \mathrm{THz}$. Weak excitation of electric dipole, $p_{y}$, and the magnetic dipole, $m_{z}$ observed. (b) Case 2: electric field in-plane and normal to the gaps, magnetic field out-of-plane; two resonances are observed, that observed in case 1 plus another one at $f_{2}=106 \mathrm{THz}$. Small overlap between the electric dipole, $p_{x}$, and the magnetic dipole, $m_{z}$ observed. (c) Case 3: electric and magnetic fields in-plane, along the gaps and normal to them, respectively; no resonances are observed. Insets show the real part of $E_{z}$ at the corresponding resonance frequencies using a rainbow colormap ( $\mathrm{min}$ in blue, zero in green, and max in red).

In Figure 5, we plot the scattering cross-sections of the 4-SRR (even) for the three cases defined in Figure 1. For this case, case 1 and case 2 are identical due to the symmetry of the 4-SRR and are referred to from here on as case 2 . In case 2 , only modes that are even with respect to the $x y$-plane and odd with respect to the $y z$-plane can be excited. In case 3, only modes that are even with respect to the $y z$-plane and odd with respect to the $x z$-plane can be excited. Due to these symmetry constraints, the electric dipole, $p_{x}$, cannot be excited in case 3 , the electric dipole, $p_{y}$, cannot be excited in case 2 , the magnetic dipole, $m_{z}$, and the electric quadrupole, $Q_{x y}$, cannot be excited in case 3 . As a result, there can only be an overlap between the electric dipole, $p_{x}$, and the magnetic dipole, $m_{z}$, in case 2 . However, as can be seen from Figure 5, only the magnetic dipole, $m_{z}$, is strongly excited in this case $\left(\mathrm{f}_{1}=203 \mathrm{THz}\right)$. For the 4-SRR, there is thus no preferred illumination scenario. 


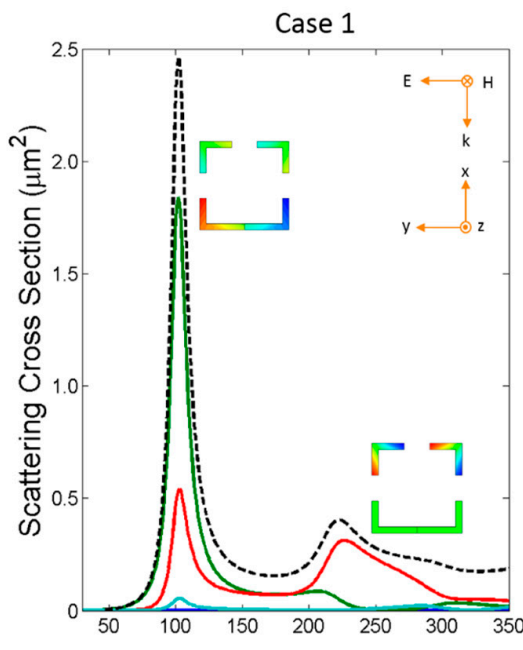

(a)

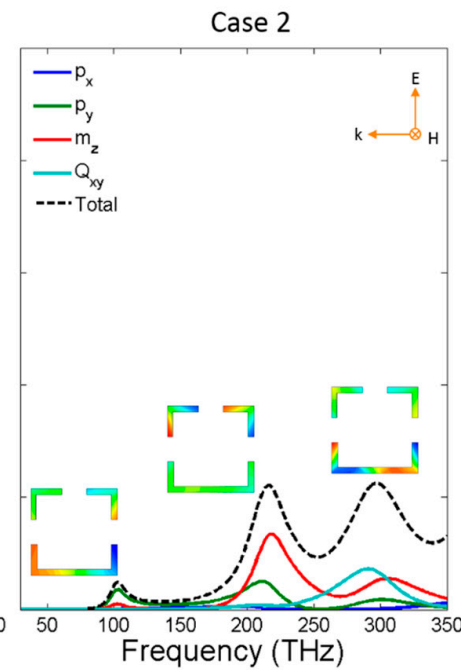

(b)

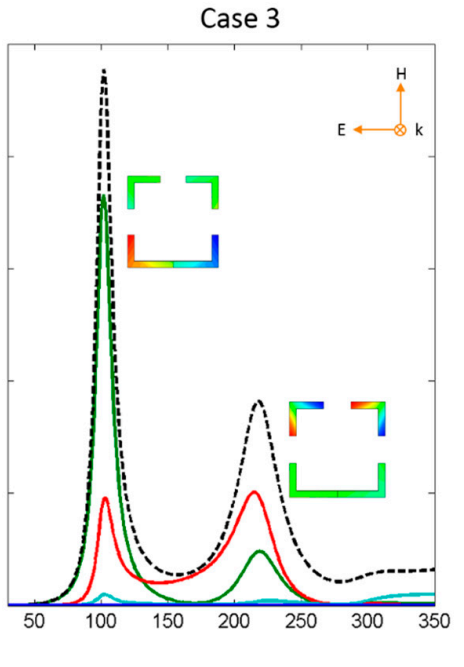

(c)

Figure 4. Multipolar decomposition of the scattering cross-section, for a 3-SRR between $30 \mathrm{THz}$ and $350 \mathrm{THz}$, into the lowest order multipoles; (a) Case 1: electric field in-plane and along the single gap, magnetic field out-of-plane; two resonances are observed at $\mathrm{f}_{1}=121 \mathrm{THz}$ and $\mathrm{f}_{2}=219 \mathrm{THz}$ (b) Case 2: electric field in-plane and normal to the single gap, magnetic field out-of-plane; three resonances are observed, those observed in case 1 plus another one at $f_{3}=297 \mathrm{THz}$ (c) Case 3: electric and magnetic fields in-plane, along the single gap and normal to it, respectively; two resonances are observed, just as in case 1. The resonances excited in Case 1 and 3 yield both an electric dipole, $p_{y}$, and a magnetic dipole, $m_{z}$, response similar to the 1-SRR. Insets show the real part of $E_{z}$ at the corresponding resonance frequencies using a rainbow colormap (min in blue, zero in green, and max in red).

Figures 2-5 help us paint a portrait of the rich multipolar response of SRRs to electromagnetic radiation. For odd-gap rings $(2 \mathrm{~N}+1-\mathrm{SRR})$, the fundamental resonance exhibits strong and overlapping electric and magnetic dipole responses regardless of the illumination, while for even-gap rings (2N-SRR), it exhibits a strong magnetic dipole response when the magnetic field is out-of-plane. Hence, the parity of the rings plays a crucial role in SRR's electromagnetic response, with analogous resonances for the odd-gap rings and even-gap rings.

We have shown that the planar configuration of SRRs, which is the one currently amenable to fabrication in the optical range, is not the best for multipole overlap. This highlights the need to move away from periodic, top-down structures and towards random, bottom-up ones. We note that this can also be achieved using dielectric nanoparticles, such as disk or ring resonators [28]. Moreover, we also note that recent works have extended investigations of randomness to the field of metasurfaces $[29,30]$. 


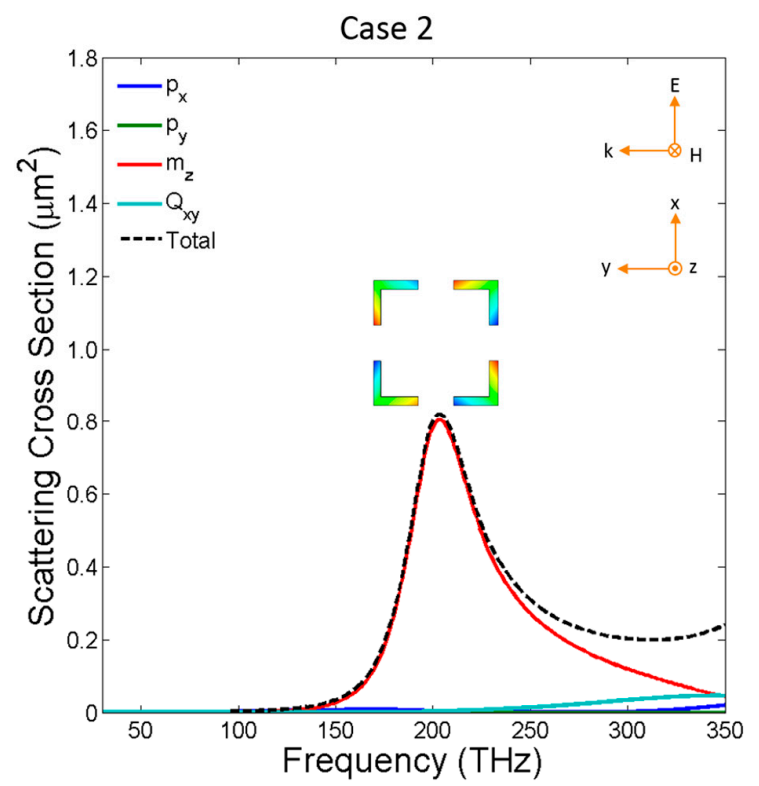

(a)

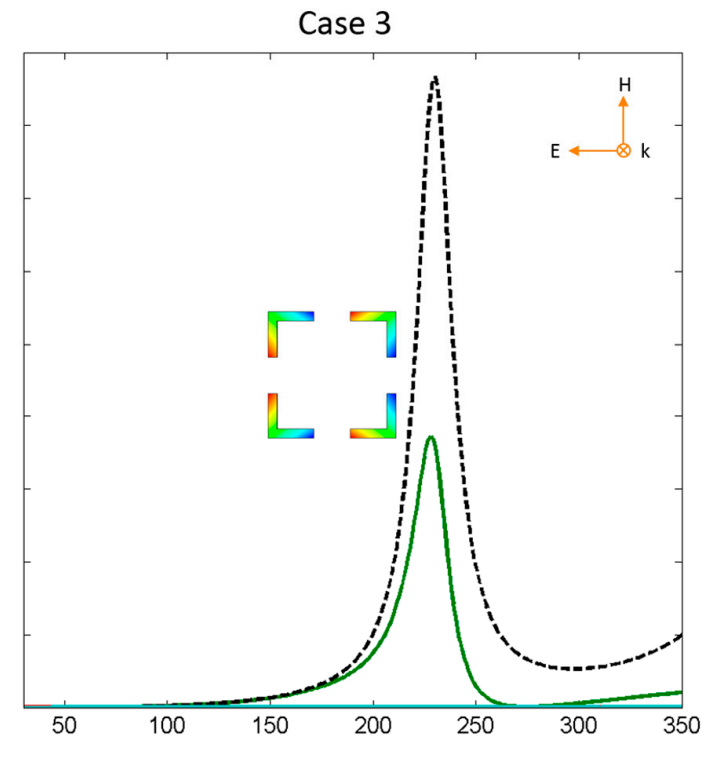

(b)

Figure 5. Multipolar decomposition of the scattering cross-section, for a 4-SRR between $30 \mathrm{THz}$ and $350 \mathrm{THz}$, into the lowest order multipoles; (a) Case 2: electric field in-plane, magnetic field out-of-plane; one resonance is observed at $f_{1}=203 \mathrm{THz}$. Strong magnetic dipole, $m_{z}$, excitation is observed. (b) Case 3: electric and magnetic fields in-plane; one resonance is observed at $\mathrm{f}_{2}=230 \mathrm{THz}$. Only electric dipole, $p_{y}$, excitation is observed. Insets show the real part of $E_{z}$ at the corresponding resonance frequencies using a rainbow colormap (min in blue, zero in green, and max in red).

\section{Conclusion}

We investigated the scattering response of SRRs with increasing gap numbers, from one to four, under three different illumination schemes. We found agreement between our results and previous works on one-gap SRRs under normal and grazing incidence and extended it to two, three, and four gaps SRRs. In a nutshell, odd-gap rings are more favorable to multipole overlap than even-gap rings and grazing incidence is more favorable than normal incidence. These findings confirm the need, especially for optics, to move towards random configurations. This could be achieved with either plasmonic or dielectric nanoparticles suspended in an aqueous solution. Furthermore, scattering properties of individual nanoparticles can be experimentally studied using techniques such as dark field microscopy. This detailed knowledge of the Cartesian multipolar response of multi-gap SRRs will accelerate the engineering of disordered metamaterials in all three dimensions.

\section{Acknowledgments}

This work was supported in part by the Office of Naval Research Multi-University Research Initiative (N00014-13-1-0678). 


\section{Author Contributions}

Andrew Chen and Thomas Lepetit implemented the algorithm, Andrew Chen and Ashok Kodigala performed the simulations, Boubacar Kanté conceived the project and supervised the research, and all authors contributed to the manuscript.

\section{Conflict of Interest}

The authors declare no conflict of interest.

\section{References}

1. Engheta, N.; Ziolkowski, R.W. Electromagnetic Metamaterials: Physics and Engineering Explorations; 1st ed.; Wiley: Hoboken, NJ, USA, 2006.

2. Shadrivov, I.V.; Lapine, M.; Kivshar Y.S. Nonlinear, Tunable and Active Metamaterials, 1st ed.; Springer: New York, NY, USA, 2015.

3. Shelby, R.A.; Smith, D.R.; Schultz, S. Experimental Verification of a Negative Index of Refraction. Science 2001, 292, 77-79.

4. Linden, S.; Enkrich, C.; Wegener, M.; Zhou, J.; Koschny, T.; Soukoulis, C.M. Magnetic Response of Metamaterials at 100 Terahertz. Science 2004, 306, 1351-1353.

5. Shalaev, V.M. Optical negative-index metamaterials. Nat. Phot. 2007, 1, 41-48.

6. Pendry, J.B.; Schurig, D.; Smith, D.R. Controlling Electromagnetic Fields. Science 2006, 312 , $1780-1782$.

7. Leonhardt, U. Optical Conformal Mapping. Science 2006, 312, 1777-1780.

8. Serdar Onses, M.; Song, C.; Williamson, L.; Sutanto, E.; Ferreira, P.M.; Alleyne, A.G.; Nealey, P.F.; Ahn, H.; Rogers, J.A. Hierarchical patterns of three-dimensional block-copolymer films formed by electrohydrodynamic jet printing and self-assembly. Nat. Nanotech. 2013, 8, 667-675.

9. Yang, S.; Ni, X.; Yin, X.; Kanté, B.; Zhang, P.; Zhu, J.; Wang, Y.; Zhang, X. Feedback-driven self-assembly of symmetry-breaking optical metamaterials in solution. Nat. Nanotech. 2014, 9, 1002-1006.

10. Gorkunov, M.V.; Gredeskul, S.A.; Shadrivov, I.V.; Kivshar, Y.S. Effect of microscopic disorder on magnetic properties of metamaterials. Phys. Rev. E 2006, 73, 056605.

11. Rockstuhl, C.; Menzel, C.; Mühlig, S.; Petschulat, J.; Helgert, C.; Etrich, C.; Chipouline, A.; Pertsch, T.; Lederer, F. Scattering properties of meta-atoms. Phys. Rev. B 2011, 83, 245119.

12. Smith, D.R.; Schultz, S.; Markoš, P.; Soukoulis, C.M. Determination of effective permittivity and permeability of metamaterials from reflection and transmission coefficients. Phys. Rev. B 2002, 65, 195104.

13. Russakoff, G. A Derivation of the Macroscopic Maxwell Equations. Am. J. Phys. 1970, 38, 1188-1195.

14. Serdyukov, A.; Semchenko, I.; Tretyakov, S.; Sihvola, A. Electromagnetics of Bi-Anisotropic Materials-Theory and Applications, 1st ed.; Gordon and Breach: Amsterdam, The Netherlands, 2001.

15. Sihvola, A. Electromagnetic Mixing Formulae and Applications, 1st ed.; IEEE Electromagnetic Waves Series: London, UK, 1999. 
16. Wiltshire, M.C.K.; Pendry, J.B.; Young, I.R.; Larkman, D.J.; Gilderdale, D.J.; Hajnal, J.V. Microstructured Magnetic Materials for RF Flux Guides in Magnetic Resonance Imaging. Science 2001, 291, 849-851.

17. Schurig, D.; Mock, J.J.; Justice, B.J.; Cummer, S.A.; Pendry, J.B.; Starr, A.F.; Smith, D.R. Metamaterial Electromagnetic Cloak at Microwave Frequencies. Science 2006, 314, 977-980.

18. Pendry, J.B.; Holden, A.J.; Robbins, D.J.; Stewart, W.J. Magnetism from Conductors and Enhanced Nonlinear Phenomena. IEEE Trans. Microw. Theory Tech. 1999, 47, 2075-2084.

19. Rockstuhl, C.; Lederer, F.; Etrich, C.; Zentgraf, T.; Kuhl, J.; Giessen, H. On the reinterpretation of resonances in split-ring resonators at normal incidence. Opt. Expr. 2006, 14, 8827-8836.

20. Kanté, B.; de Lustrac, A.; Lourtioz, J.-M.; Gadot, F. Engineering resonances in infrared metamaterials. Opt. Expr. 2008, 16, 6774-6784.

21. Chen, C.-Y.; Wu, S.-C.; Yen, T.-J. Experimental verification of standing-wave plasmonic resonances in split-ring resonators. Appl. Phys. Lett. 2008, 93, 034110.

22. Penciu R.S.; Aydin K.; Kafesaki M.; Koschny T.; Ozbay E.; Economou E.N.; Soukoulis, C.M. Multi-gap individual and coupled split-ring resonator structures. Opt. Expr. 2008, 18131-18144.

23. Zhou, J.; Koschny, T.; Kafesaki, M.; Economou, E.N.; Pendry, J.B.; Soukoulis, C.M. Saturation of the Magnetic Response of Split-Ring Resonators at Optical Frequencies. Phys. Rev. Lett. 2005, 95, 223902.

24. Chen, C.-Y.; Yen, T.-J. Electric and magnetic responses in the multiple-split ring resonators by electric excitation. J. Appl. Phys. 2009, 105, 124913.

25. Kanté, B.; O’Brien, K.; Niv, A.; Yin, X.; Zhang, X. Proposed isotropic negative index in threedimensional optical metamaterials. Phys. Rev. B 2012, 85, 041103(R).

26. Mühlig, S.; Menzel, C.; Rockstuhl, C.; Lederer, F. Multipole analysis of meta-atoms. Metamaterials 2010, 5, 64-73.

27. Bohren, C.F.; Huffman, D.R. Absorption and Scattering of Light by Small Particles, 1st ed.; Wiley: Weinheim, Germany, 2004.

28. Lepetit, T.; Kanté, B. Controlling multipolar radiation with symmetries for electromagnetic bound states in the continuum. Phys. Rev. B 2014, 90, 241103(R).

29. Albooyeh, M; Morits, D.; Tretyakov, S.A. Effective electric and magnetic properties of metasurfaces in transition from crystalline to amorphous state. Phys. Rev. B 2012, 85, 205110.

30. Albooyeh, M.; Kruk, S.; Menzel, C.; Helgert, C.; Kroll, M.; Krysinski, A.; Decker, M.; Neshev, D.N.; Pertsch, T.; Etrich, C.; Rockstuhl, C.; Tretyakov, S.A.; Simovski, C.R.; Kivshar, Y.S. Resonant metasurfaces at oblique incidence: Interplay of order and disorder. Sci. Rep. 2014, 4, 4484.

31. Johnson, P.B.; Christy, R.W. Optical constants of the noble metals. Phys. Rev. B 1972, 6, 4370.

(C) 2015 by the authors; licensee MDPI, Basel, Switzerland. This article is an open access article distributed under the terms and conditions of the Creative Commons Attribution license (http://creativecommons.org/licenses/by/4.0/). 